\title{
Identification of QTNs Controlling 100-Seed Weight in Soybean Using Multilocus Genome-Wide Association Studies
}

\author{
Zhongying Qi', Jie Song ${ }^{1}$, Kaixin Zhang ${ }^{1}$, Shulin Liu' ${ }^{2}$, Xiaocui Tian', Yue Wang ${ }^{1}$, \\ Yanlong Fang ${ }^{1}$, Xiyu Li ${ }^{1}$, Jiajing Wang ${ }^{1}$, Chang Yang ${ }^{1}$, Sitong Jiang ${ }^{1}, X u$ Sun ${ }^{1}$, \\ Zhixi Tian', Wenxia Lit* $^{1 *}$ and Hailong Ning ${ }^{1 *}$ \\ 1 Key Laboratory of Soybean Biology, Ministry of Education, Key Laboratory of Soybean Biology and Breeding/Genetics, \\ Ministry of Agriculture, Northeast Agricultural University, Harbin, China, ${ }^{2}$ State Key Laboratory of Plant Cell and Chromosome \\ Engineering, Institute of Genetics and Developmental Biology, Chinese Academy of Sciences, Beijing, China
}

OPEN ACCESS

Edited by:

Genlou Sun,

Saint Mary's University, Canada

Reviewed by:

Yuan-Ming Zhang,

Huazhong Agricultural University,

China

Zhenyu Jia,

University of California, Riverside,

United States

Liu Jin Dong,

Chinese Academy of Agricultural

Sciences, China

*Correspondence:

Wenxia Li

liwenxianeau@126.com

Hailong Ning

ninghailongneau@126.com

Specialty section:

This article was submitted to Evolutionary and Population Genetics,

a section of the journal

Frontiers in Genetics

Received: 26 October 2019

Accepted: 04 June 2020

Published: 16 July 2020

Citation:

Qi Z, Song J, Zhang K, Liu S, Tian $X$, Wang $Y$, Fang $Y, L i X$, Wang J, Yang $C$, Jiang $S$, Sun $X$, Tian Z, Li W and Ning $H$ (2020) Identification of QTNs Controlling 100-Seed Weight in Soybean Using Multilocus

Genome-Wide Association Studies.

Front. Genet. 11:689.

doi: $10.3389 /$ fgene.2020.00689
Hundred-seed weight (HSW) is an important measure of yield and a useful indicator to monitor the inheritance of quantitative traits affected by genotype and environmental conditions. To identify quantitative trait nucleotides (QTNs) and mine genes useful for breeding high-yielding and high-quality soybean (Glycine max) cultivars, we conducted a multilocus genome-wide association study (GWAS) on HSW of soybean based on phenotypic data from 20 different environments and genotypic data for 109,676 singlenucleotide polymorphisms (SNPS) in 144 four-way recombinant inbred lines. Using five multilocus GWAS methods, we identified 118 QTNs controlling HSW. Among these, 31 common QTNs were detected by various methods or across multiple environments. Pathway analysis identified three potential candidate genes associated with HSW in soybean. We used allele information to study the common QTNs in 20 large-seed and 20 small-seed lines and identified a higher percentage of superior alleles in the largeseed lines than in small-seed lines. These observations will contribute to construct the gene networks controlling HSW in soybean, which can improve the genetic understanding of HSW, and provide assistance for molecular breeding of soybean large-seed varieties.

Keywords: soybean, hundred-seed weight, multilocus GWAS, QTNs, four-way RILs

\section{INTRODUCTION}

Soybean [Glycine max (L.) Merr.] is an important source of edible oil and plant protein for humans. Soy-based foods are popular in the international market, and the demand for soybeans is increasing. Hundred-seed weight (HSW) is an important agronomic trait related to soybean yield and food quality. Therefore, locating the quantitative trait locus (QTLs) related to HSW and exploring valuable alleles associated with the QTLs have important theoretical and application value for improving soybean yield and quality.

Linkage analysis based on framework linkage maps of low polymorphism molecular markers, such as restriction fragment length polymorphisms (RFLPs) and simple sequence repeats (SSRs), is the main method for detecting QTLs controlling HSW in soybean. Quantitative trait loci associated with HSW have been identified in $F_{2}$ (Wang et al., 2010), recombinant inbred line (RIL; Reinprecht et al., 2006), and backcross (BC; Li W.X. et al., 2008) populations 
using QTL mapping methods such as interval mapping (IM; Lander and Botstein, 1989), composite interval mapping (CIM; Zeng, 1994), and inclusive complete interval mapping (Wang, 2009). Genome-wide CIM (Wang et al., 2016b; Wen et al., 2017) was recently proposed for detecting small-effect and linked QTLs. However, QTLs detected by these methods have rarely been applied successfully to marker-assisted breeding research because large linkage between markers and QTL is feasible to be interrupted. Thus, genome-wide association studies (GWASs) have been conducted gradually for QTL mapping of HSW in soybean.

Drifting and selection of genes during crop breeding and domestication produce complex population structures, which can cause false positives. Statistical methods to circumvent this issue include case-control studies, transmission imbalance tests, structured associations, principal component analysis (PCA), and hybrid models. A newly developed GWAS statistical method based on a mixed linear model (MLM) can overcome the above difficulties and address fixed and random effects flexibly. Common variables from STRUCTURE (Pritchard et al., 2000) and PCA can serve as fixed effects to account for false associations due to group structure. The complex relationship between individuals constitutes the affinity matrix in the MLM (Yu et al., 2006). The kinship matrix derived from a set of aggregated individuals is used in the computationally efficient compressed MLM (CMLM; Zhang et al., 2010). In some cases, the traditional maximum-likelihood method cannot be used to solve an MLM with a large number of genotypes because the calculation intensity is too large. Therefore, the efficient mixed model association (EMMA; Kang et al., 2008) algorithm was developed to reset the parameter of MLM likelihood function. Among the many software packages available, mrMLM.GUI integrates the most accurate and computationally efficient methods for GWAS and genome prediction selection, and these methods are integrated into the $\mathrm{R}$ package, which can analyze large amounts of data in the shortest time.

Genome-wide association study has been used to detect QTLs related to HSW in soybean. Zhou et al. (2015) used a total of 302 germplasm accessions including wild soybean varieties, local varieties, and bred cultivars to detect HSW loci on chromosome 17, while Zhang et al. (2016) used 309 soybean germplasm accessions to detect 22 HSW QTLs by GWAS analysis. These studies used single-gene locus methods such as MLM (Yu et al., 2006; Zhang et al., 2010). The mrMLM method used in our study is a multilocus method that detects more small-effect QTLs than the single-locus method (He et al., 2019), reducing the probability of false positives (Fang et al., 2017).

We used 144 RILs with four-way hybridization (FW-RILs) to obtain phenotypic data for the HSW and SNP genotype data across multiple environments. The multilocus GWAS method was used to detect quantitative trait nucleotides (QTNs) associated with HSW. We identified potential candidate genes and common QTNs associated with large-seed lines across multiple methods or in multiple environments, laying the foundation for high-yield soybean breeding.

\section{MATERIALS AND METHODS}

\section{Plant Materials}

In 2008, the four soybean varieties Kenfeng 14 (HSW 21 g), Kenfeng 15 (HSW 18 g), Kenfeng 19 (HSW 19 g), and Heinong 48 (HSW 25 g) were used to prepare double hybrid combinations. Kenfeng 14, Kenfeng 15, Kenfeng 19, and Heinong 48 were derived from the crosses Suinong $10 \times$ Changnong 5, Suinong $14 \times$ Kenjiao 9307, Hefeng $25 \times$ (Kenfeng $4 \times$ Gong 8861-0), and Ha 90-6719 $\times$ Sui 90-5888, respectively. In 2009, $\mathrm{F}_{1}$ (Kenfeng $14 \times$ Kenfeng 15) was used as the female parent, and $\mathrm{F}_{1}$ (Kenfeng $19 \times$ Heinong 48) was used as the male parent to produce fourway hybrid $F_{1}$ seeds. From 2010 to 2012, the seeds were planted in Harbin in the summer and in Hainan in the winter. Four-way RILs (144 lines) were obtained by continuous self-crossing for six generations using the single-seed descent method.

\section{Field Experiments and Phenotypic Data Collection}

The 144 FW-RILs were planted in 20 environments; details of the planting environments are summarized in Supplementary Table S1. The field experiment in each environment followed a randomized block design. Plot length was $5 \mathrm{~m}$, ridge distance was $65 \mathrm{~cm}$, plant spacing was $6 \mathrm{~cm}$, three rows were repeated three times, and field management was in accordance with general field cultivation. After maturity, 10 plants showing uniform growth were selected from each row, and HSW was measured indoors. Phenotypic values are all averages of three replicates.

\section{Phenotypic Variation Analysis}

Mean, variance, standard deviation, minimum, maximum, skewness, and kurtosis of FW-RILs were calculated in each environment. Analysis of variance was performed on phenotypic data from the 20 environments using the following model:

$$
x_{i j}=\mu+G_{i}+E_{j}+G E_{i j}+\varepsilon_{i j}
$$

where $\mu$ is the grand average, $G_{i}$ is the $i$ th genotype effect, $E_{j}$ is the $j$ th environment effect, $G E_{i j}$ is the genotype $\times$ environmental effect, and $\varepsilon_{i j}$ is the error effect following $\mathrm{N}\left(0, \sigma^{2}\right)$.

The mean squared difference was estimated by applying the mean square of each variation source, and the estimated generalized heritability was calculated using the following formula.

$$
h^{2}=\frac{\sigma_{G}^{2}}{\sigma_{G}^{2}+\frac{\sigma_{G E}^{2}}{e}+\frac{\sigma^{2}}{e r}}
$$

where $h^{2}$ is the generalized heritability of gene $\times$ environment interaction, $\sigma_{G}^{2}$ is the genotype variance, $\sigma_{G E}^{2}$ is the genotype $\times$ environmental interaction variance, $\sigma^{2}$ is the error variance, $e$ is the number of environments, and $r$ is the number of repetitions in each environment. Data were analyzed using the general linear model method in Statistical Analysis System (SAS) 9.2, North Carolina.

\section{Genotyping}

Leaves of each accession were collected in the field at the three-leaf stage and placed in $2-\mathrm{mL}$ centrifuge tubes. Liquid 
TABLE 1 | Joint ANOVA of HSW of FW-RILs in multiple environments and heritability.

\begin{tabular}{|c|c|c|c|c|c|c|}
\hline Source & DF & ss & MS & $\boldsymbol{F}$ & $\operatorname{Pr}>F$ & Variance component \\
\hline Replication & 2 & 0.0060 & 0.0030 & 1.43 & 0.2390 & - \\
\hline Environment & 19 & $12,262.04$ & 645.37 & 308,403 & $<0.0001$ & 1.72 \\
\hline Genotype & 143 & $10,032.07$ & 70.15 & $33,524.6$ & $<0.0001$ & 1.02 \\
\hline Genotype $\times$ environment & 2,385 & $38,569.94$ & 16.17 & $7,728.05$ & $<0.0001$ & 5.39 \\
\hline Error & 5,094 & 10.66 & 0.0021 & - & - & 0.0021 \\
\hline$h^{2}$ & - & - & - & - & - & 0.79 \\
\hline
\end{tabular}

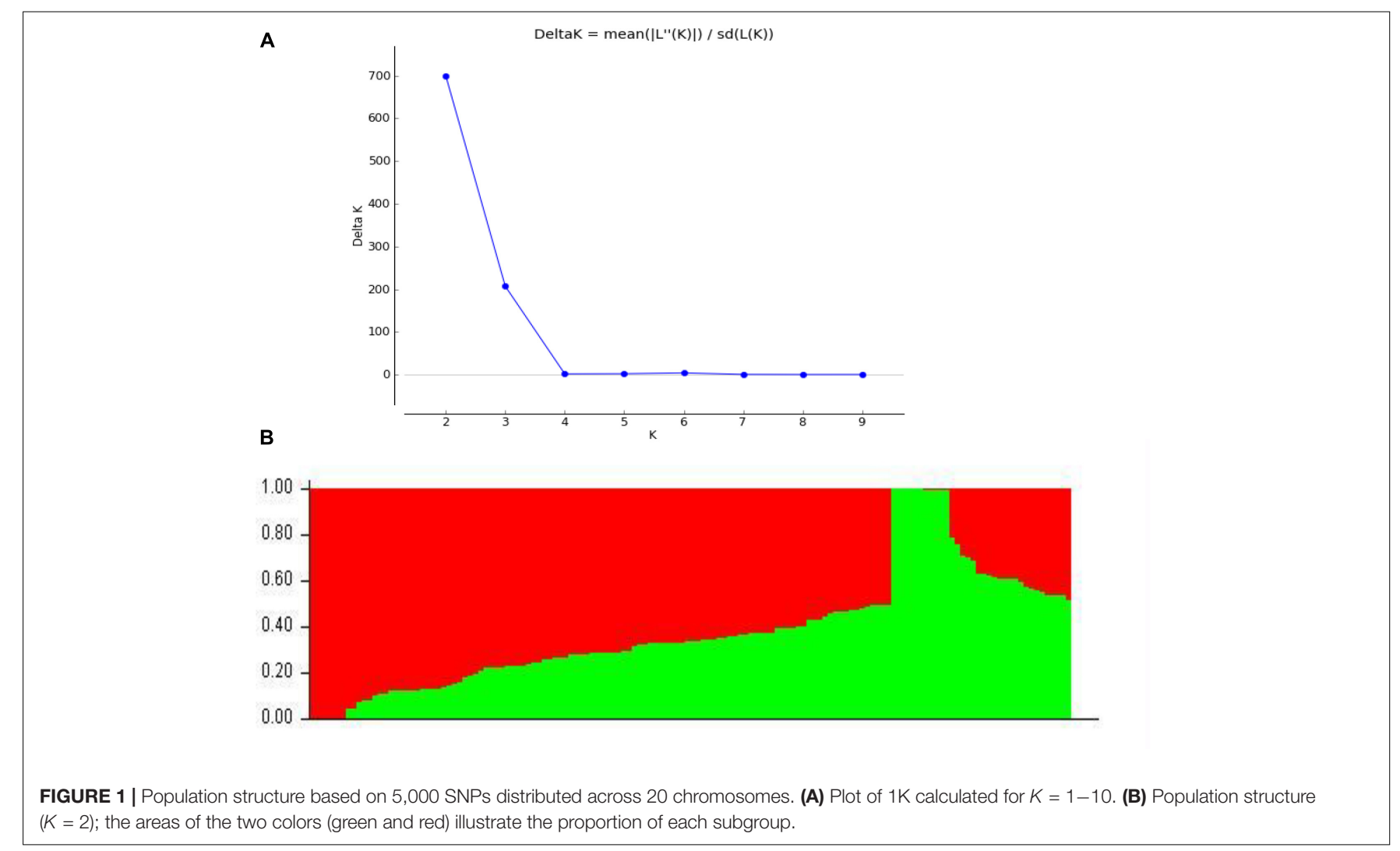

nitrogen was added to the tubes, and leaves were rapidly ground to a white powder. Soybean genomic DNA was extracted using the CTAB method (Doyle et al., 1990). DNA was dissolved

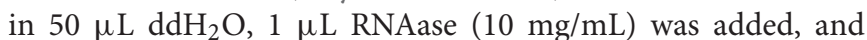
samples were stored at $-20^{\circ} \mathrm{C}$. Single-nucleotide polymorphism genotyping was performed by Beijing Boao Biotechnology Co., Ltd. (Beijing, China) using the SoySNP660K BeadChip. A total of 109,676 SNPs were obtained on 20 chromosomes after mass filtration based on the following criteria: minor allele frequency (MAF) of the SNP was identified (MAF > 0.05), maximum missing data rate $<10 \%$ (Belamkar et al., 2016), and heterozygous loci deleted.

\section{Analysis of Linkage Disequilibrium and Population Structure}

Linkage disequilibrium (LD) analysis was performed using TASSEL 5.0 (Bradbury et al., 2007) to calculate the square of the allelic frequency dependence $\left(r^{2}\right)$ of all SNPs located within $10-\mathrm{Mb}$ physical distance, and the physical distance of LD decay was estimated as the position where $r^{2}$ dropped to half of its maximum value.

Population structure was analyzed after filtration on 5,000 SNPs evenly distributed on 20 chromosomes using STRUCTURE 2.3.4 (Pritchard et al., 2000). The number of burn-in iterations per run was 100,000, followed by 100,000 Markov Chain Monte Carlo replications after burn-in. Mixed and allele frequency correlation models were considered in the analysis. Five implicit iterations were used in the STRUCTURE analysis. The best subgroups (K) were identified according to the method of Evanno et al. (2005) using STRUCTURE HARVESTER (Earl and Vonholdt, 2012). The assumed number of $K$ ranged from 1 to 10 .

\section{Genome-Wide Association Studies}

Genome-wide association studies were performed using the software mrMLM.GUI (version 3.0). There are five multilocus GWAS methods in the mrMLM package that can be used to identify significant QTNs, namely, mrMLM (Wang et al., 2016a), FASTmrMLM (Tamba and Zhang, 2018), FASTmrEMMA 


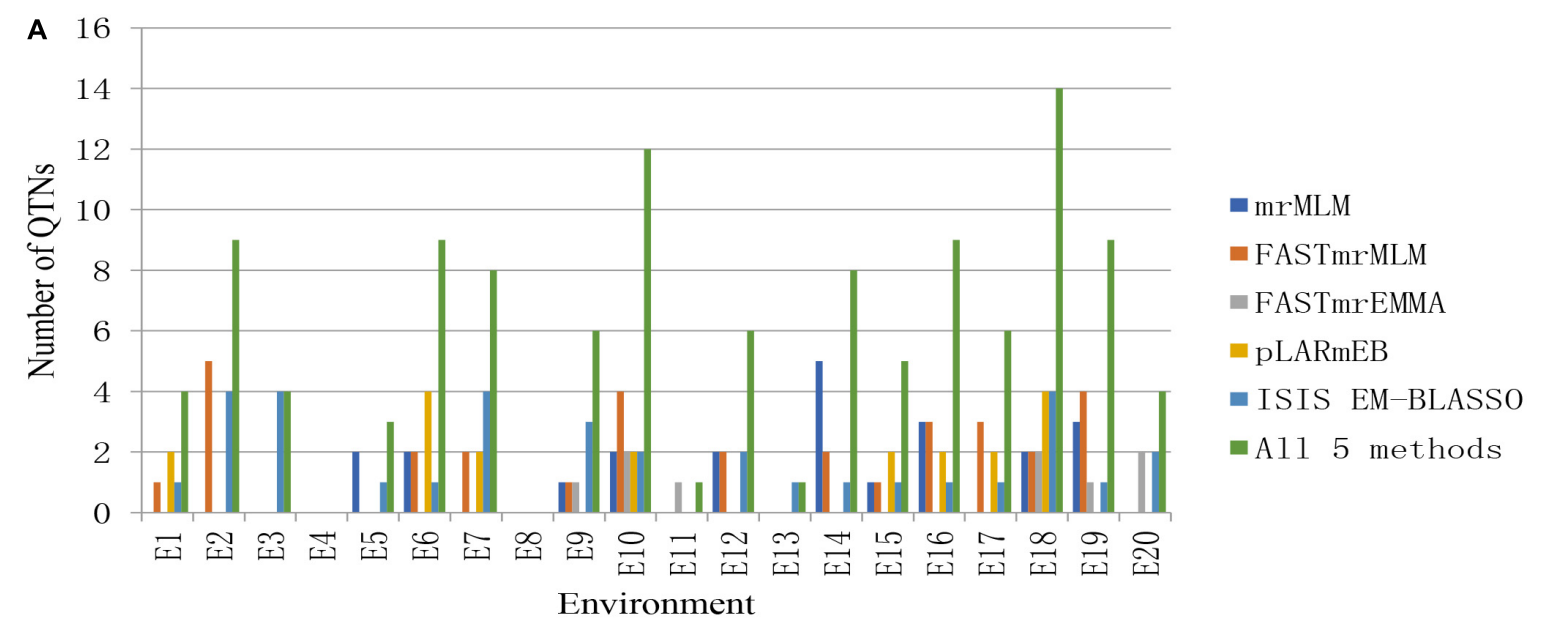

B

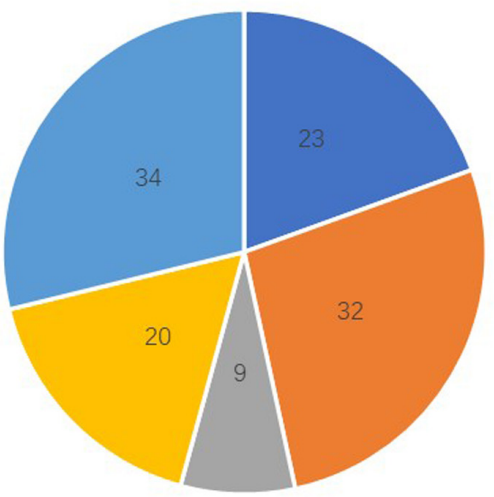

- mrMLM = FASTmrMLM = FASTmrEMMA = pLARmEB -ISIS EM-BLASSO

FIGURE 2 | (A) The total numbers of significant QTNs detected in 20 environments across five methods. (B) The total numbers of significant QTNs detected using each of five multilocus GWAS methods in 20 environments.

TABLE 2 | Stable expressed QTNs identified in multiple environments and by multiple methods.

\begin{tabular}{|c|c|c|c|c|c|c|c|}
\hline Method $^{\mathrm{a}}$ & Env & Marker & Chr & Marker position & QTN effect & LOD score & $r^{2}(\%)^{b}$ \\
\hline 2,5 & E2, E2 & - & - & - & $0.66,0.92$ & $3.08,4.48$ & $3.28,6.43$ \\
\hline 2,5 & E2, E3 & $A X-157369770$ & 11 & 32617288 & $-0.87,-0.75$ & $3.80,3.07$ & $5.02,3.78$ \\
\hline 2,5 & E2, E3 & $A X-157588392$ & 12 & 17219655 & $-1.01,-0.94$ & $4.20,4.26$ & $6.64,6.36$ \\
\hline
\end{tabular}

${ }^{a}$ mrMLM, FASTmrMLM, FASTMrEMMA, pLARMEB, and ISIS EM-BLASSO were indicated by $1-5$, respectively. ${ }^{2}{ }^{2}$ (\%), proportion of total phenotypic variation explained by each QTN.

(Wen et al., 2018), pLARmEB (Zhang J. et al., 2017; Zhang Y. et al., 2017), and ISIS EM-BLASSO (Tamba et al., 2017). In the first stage, the critical $P$ value parameter was set to 0.01 , except that of FASTmrEMMA was set to 0.005. In the final stage, the critical LOD value of significant QTNs was set to 3. All matrices involved in these five methods were calculated using mrMLM.GUI3.0.

\section{Superior Allele Analysis}

Among the significant QTNs identified, some were detected in a variety of environments or methods. These significant
QTNs are referred to as common QTNs. The positive and negative of the QTN effect values were used as the criterion for selecting superior alleles. If the QTN effect value is positive, the genotype of code 1, which was obtained by GWAS, is the superior allele; if the QTN effect value is negative, the other genotype is the superior allele. The percentage of excellent alleles is calculated as follows: for each line, the percentage of excellent alleles is the number of common QTNs carrying excellent alleles divided by the total number of common QTNs; for each common QTN, the percentage of alleles among the $144 \mathrm{FW}$-RILs is equal to the number of rows 
TABLE 3 | Common QTNs for hundred-seed weight in soybean across different multilocus method.

\begin{tabular}{|c|c|c|c|c|c|c|}
\hline Methoda $^{a}$ & Marker & Chromosome & Position (bp) & QTN effect & LOD score & $r^{2}(\%)^{b}$ \\
\hline $1,2,3,4,5$ & $A X-116905453$ & 2 & 44205396 & $\begin{array}{c}1.43,1.23,2.23,1.13 \\
1.15\end{array}$ & $\begin{array}{c}5.43,5.43,4.64,5.26 \\
5.48\end{array}$ & $\begin{array}{c}15.07,12.14,8.42 \\
10.12,10.74\end{array}$ \\
\hline 3,5 & $A X-157257435$ & 2 & 43283682 & $2.05,0.70$ & $3.32,3.22$ & $12.83,5.91$ \\
\hline $1,2,5$ & $A X-157170687$ & 3 & 1393672 & $0.72,0.56,0.54$ & $3.08,3.98,3.30$ & $12.40,7.52,6.94$ \\
\hline $1,4,5$ & $A X-157052803$ & 3 & 1722938 & $0.99,0.90,0.86$ & $4.38,4.95,3.86$ & $12.96,10.80,10.08$ \\
\hline 1,2 & $A X-157407598$ & 3 & 39988963 & $0.90,0.61$ & $3.52,3.52$ & $9.03,4.59$ \\
\hline $1,2,4,5$ & $A X-116924879$ & 3 & 5093950 & $\begin{array}{c}-0.82,-0.60,-0.60 \\
-0.73\end{array}$ & $3.16,3.70,4.13,4.33$ & $11.33,6.23,6.23,8.96$ \\
\hline 2,5 & $A X-157528951$ & 3 & 6197954 & $-0.66,-0.94$ & $3.51,6.68$ & $5.84,11.04$ \\
\hline $1,2,3,4,5$ & $A X-157442983$ & 4 & 6484678 & $\begin{array}{c}1.15,0.88,1.98,0.95 \\
0.92\end{array}$ & $\begin{array}{c}4.00,3.02,3.38,3.56 \\
3.77\end{array}$ & $\begin{array}{c}12.52,7.33,10.44 \\
8.03,7.98\end{array}$ \\
\hline $1,2,4,5$ & $A X-157126994$ & 6 & 49959758 & $\begin{array}{c}-0.99,-0.83,-0.78 \\
-0.64\end{array}$ & $5.67,5.67,5.31,3.87$ & $\begin{array}{c}14.03,10.74,9.52 \\
6.35\end{array}$ \\
\hline 2,5 & $A X-157525995$ & 6 & 51088854 & $0.92,0.66(0.74)$ & 4.48, 3.08 (3.90) & $6.43,3.28(4.24)$ \\
\hline $1,2,5$ & $A X-157457247$ & 7 & 3254514 & $-0.71,-0.49,-0.52$ & $4.81,3.23,3.66$ & $10.00,5.06,5.72$ \\
\hline 2,5 & $A X-157395672$ & 7 & 37249194 & $-0.77,-0.82$ & $3.67,4.26$ & $4.49,5.13$ \\
\hline 1,2 & $A X-157388275$ & 8 & 46069989 & $-0.88,-0.70$ & $4.08,4.08$ & $11.96,7.74$ \\
\hline 1,2 & $A X-157555217$ & 8 & 47740465 & $0.95,0.87$ & $3.85,4.22$ & $13.46,11.42$ \\
\hline 4,5 & $A X-157129457$ & 9 & 40507825 & $0.59,0.59$ & $3.71,3.13$ & $3.80,3.82$ \\
\hline $1,2,3,4,5$ & $A X-157355369$ & 10 & 49273669 & $\begin{array}{c}0.90,0.63,1.25,0.70 \\
0.56\end{array}$ & $\begin{array}{c}3.52,3.52,3.70,5.02 \\
3.00\end{array}$ & $\begin{array}{c}12.43,6.50,6.00 \\
8.03,5.19\end{array}$ \\
\hline 1,2 & $A X-157480162$ & 10 & 50172467 & $0.85,0.60$ & $3.14,3.12$ & $8.82,4.66$ \\
\hline 2,5 & $A X-157369770$ & 11 & 32617288 & $-0.87,-0.75$ & $3.80,3.07$ & $5.02,3.78$ \\
\hline 2,5 & $A X-157588392$ & 12 & 17219655 & $-1.01,-0.94$ & $4.20,4.26$ & $6.64,6.36$ \\
\hline 3,5 & $A X-157090659$ & 14 & 881999 & $1.57,0.80$ & $3.28,3.01$ & $4.80,5.21$ \\
\hline 3,5 & $A X-157446872$ & 14 & 37252443 & $-1.75,-0.88$ & $3.86,4.15$ & $6.61,7.80$ \\
\hline $2,3,4$ & $A X-157439837$ & 15 & 12672673 & $1.16 \mathrm{E}-05,1.01,0.46$ & $3.60,3.45,3.22$ & 3.53E-09, 6.34, 5.15 \\
\hline 1,2 & $A X-157069540$ & 16 & 37223445 & $-0.67,-0.55$ & $4.17,4.17$ & $10.06,6.91$ \\
\hline 2,5 & $A X-157575017$ & 16 & 32946305 & $-0.43,-0.45$ & $3.06,3.24$ & $4.35,4.78$ \\
\hline $1,2,4$ & $A X-157516116$ & 16 & 33525379 & $-0.72,-0.55,-0.55$ & $3.35,4.44,5.65$ & $10.30,6.08,6.09$ \\
\hline 2,4 & $A X-157553398$ & 18 & 56507541 & $0.48,0.49$ & $3.52,3.05$ & $4.43,4.63$ \\
\hline $1,2,4$ & $A X-157268182$ & 18 & 56589017 & $-0.81,-0.60,-0.54$ & $3.89,3.89,3.48$ & $9.95,6.05,4.92$ \\
\hline 1,2 & $A X-157352830$ & 18 & 56836743 & $0.89,0.71$ & $3.75,4.55$ & $11.44,7.76$ \\
\hline 1,2 & $A X-157467282$ & 20 & 43174088 & $-0.77,-0.43$ & $4.71,3.67$ & $11.61,3.93$ \\
\hline 1,2 & $A X-116884145$ & 20 & 47162277 & $-0.75,-0.50$ & $3.19,3.01$ & $8.45,3.94$ \\
\hline 2,5 & $A X-157324617$ & 20 & 42347884 & $0.54,0.66$ & $3.31,3.73$ & $5.35,7.38$ \\
\hline
\end{tabular}

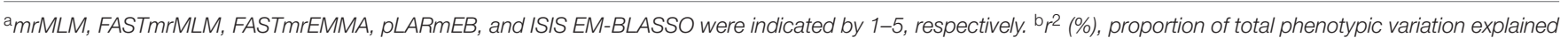
by each QTN. Bold text indicates the QTNs appeared to be near QTLs associated with hundred-seed weight in soybean that had been mapped in earlier studies.

containing the alleles divided by the total number of rows. Heat maps were generated using the $\mathrm{R}$ package Complexheatmap (Mellbye and Schuster, 2014).

\section{Identification of Potential Candidate Genes}

The physical locations of generic QTNs identified by the above methods were marked. Intervals containing each of the common QTNs were then selected on the Phytozome website. These intervals are determined by the LD decay rate. Highly expressed genes at certain locations based on relevant traits were selected. This step was done on the BAR website. Finally, the genes were matched to those in the Kyoto Gene and Genomics Encyclopedia (KEGG) to analyze gene expression pathways and identify potential candidate genes.

\section{RESULTS}

\section{Phenotypic Variation}

We measured HSW phenotypic data for 144 FW-RILs and the four parent lines in 20 environments. The phenotypic values for the four parents are given in Supplementary Table S2. In 19 environments, the kurtosis and skewness (absolute value) were less than 1, indicating a continuous normal distribution of HSW (Supplementary Table S3 and Figure S1). We used SAS software to perform a descriptive statistical analysis, conduct an analysis of variance, and estimate the generalized heritability of phenotypic values (Table 1). The results showed that significant phenotypic variation was detected in the HSW of 144 FW-RILs across 20 environments. An analysis of variance revealed significant differences in genotype effects, environmental effects, and genotype $\times$ environment interactions (Table 1). Therefore, 


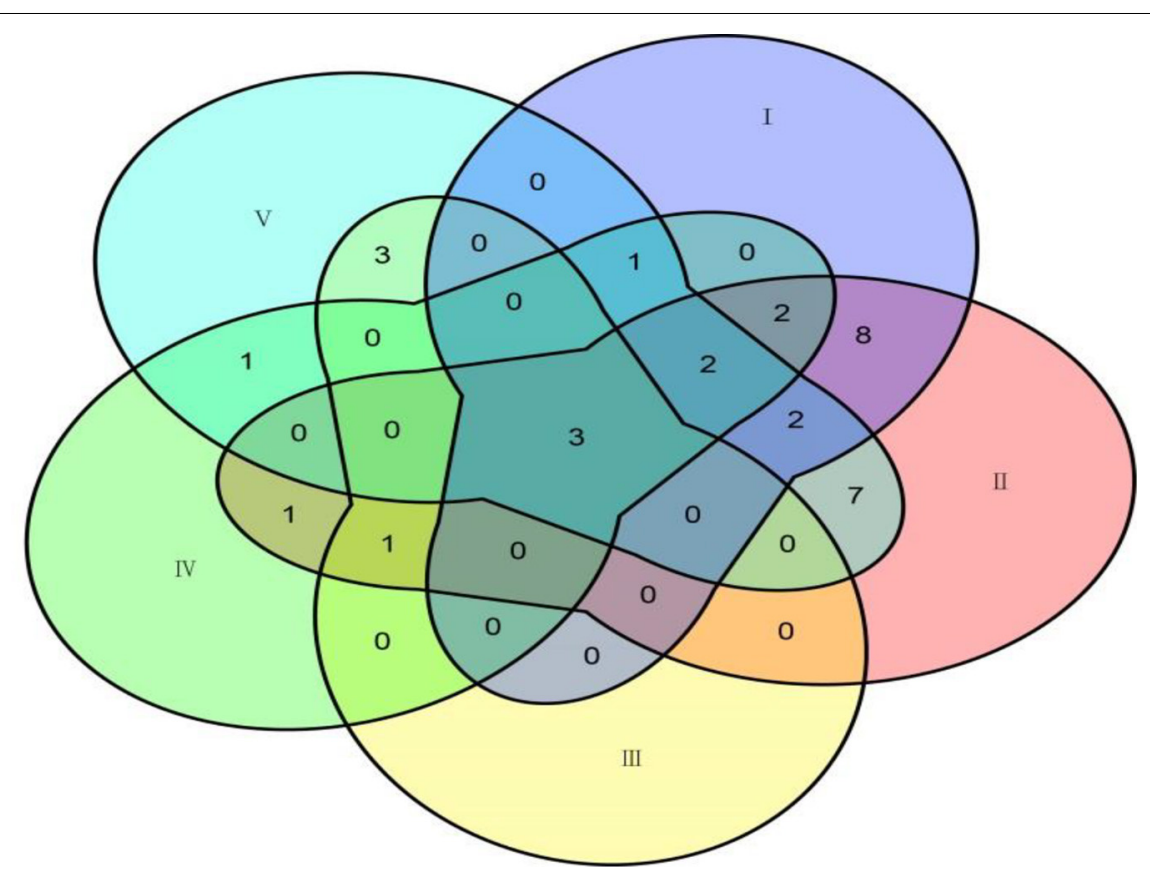

FIGURE 3 | The connection of common QTNs detected by five methods. Method numbers correspond to (I) mrMLM, (II) FASTmrMLM, (III) FASTmrEMMA, (IV) pLARmEB, and (V) ISIS EM-BLASSO.
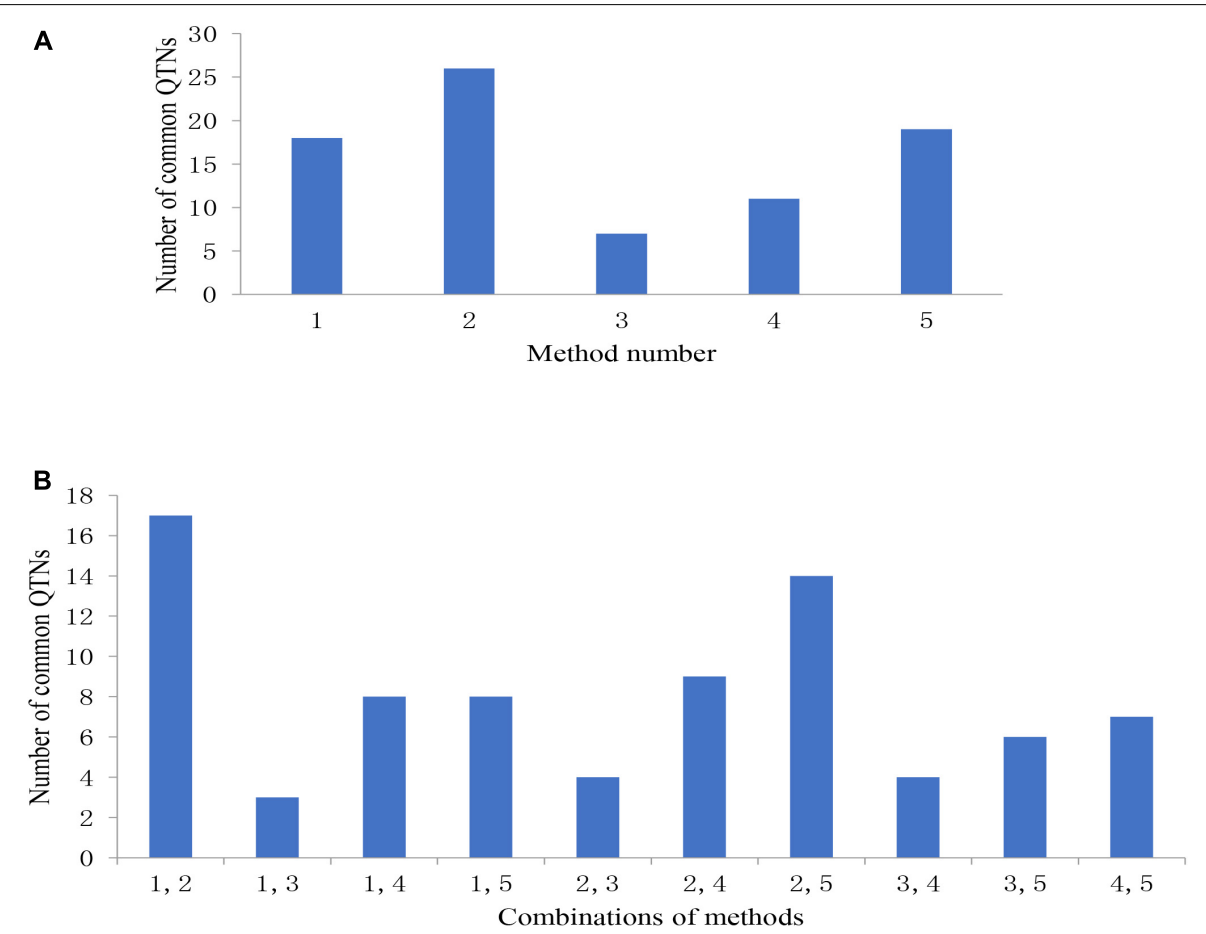

FIGURE 4 | (A) The number of common QTNs detected by different methods and (B) different combinations of methods. Method numbers correspond to (1) mrMLM, (2) FASTmrMLM, (3) FASTmrEMMA, (4) pLARmEB, and (5) ISIS EM-BLASSO.

HSW is not only affected by genotype and environmental factors, but also by genotype-by-environmental interaction effects. The heritability of HSW in multiple environments was $79 \%$. This indicates that although HSW is affected by the environment, most of the variation is due to genetic effects. 
TABLE 4 | Phenotypic averages of hundred-seed weight and proportion of superior alleles in 40 lines across 31 common QTNs.

\begin{tabular}{lccccc}
\hline Line & HSW (g) & PSA (\%) & Line & HSW (g) & PSA (\%) \\
\hline HN105 & 22.55 & 45 & HN148 & $\mathbf{1 7 . 7 9}$ & $\mathbf{3 5}$ \\
HN26 & 21.82 & 52 & HN32 & $\mathbf{1 7 . 7 8}$ & $\mathbf{3 5}$ \\
HN36 & 21.30 & 48 & HN20 & $\mathbf{1 7 . 7 7}$ & $\mathbf{3 5}$ \\
HN103 & 21.25 & 65 & HN86 & $\mathbf{1 7 . 6 9}$ & $\mathbf{3 9}$ \\
HN118 & 21.22 & 60 & HN81 & $\mathbf{1 7 . 6 8}$ & $\mathbf{4 2}$ \\
HN3 & 21.14 & 42 & HN53 & $\mathbf{1 7 . 5 2}$ & $\mathbf{3 9}$ \\
HN143 & 21.12 & 45 & HN145 & $\mathbf{1 7 . 5 1}$ & $\mathbf{3 9}$ \\
HN123 & 20.97 & 48 & HN112 & $\mathbf{1 7 . 5 0}$ & $\mathbf{2 9}$ \\
HN134 & 20.89 & 52 & HN50 & $\mathbf{1 7 . 4 4}$ & $\mathbf{3 5}$ \\
HN16 & 20.83 & 42 & HN85 & $\mathbf{1 7 . 4 3}$ & $\mathbf{4 2}$ \\
HN98 & 20.82 & 69 & HN109 & $\mathbf{1 7 . 2 9}$ & $\mathbf{5 2}$ \\
HN135 & 20.80 & 58 & HN62 & $\mathbf{1 7 . 2 4}$ & $\mathbf{3 2}$ \\
HN84 & 20.79 & 69 & HN69 & $\mathbf{1 7 . 1 9}$ & $\mathbf{3 9}$ \\
HN49 & 20.75 & 35 & HN72 & $\mathbf{1 7 . 1 5}$ & $\mathbf{3 9}$ \\
HN96 & 20.74 & 71 & HN136 & $\mathbf{1 7 . 1 1}$ & $\mathbf{3 4}$ \\
HN61 & 20.74 & 48 & HN113 & $\mathbf{1 7 . 0 8}$ & $\mathbf{2 1}$ \\
HN95 & 20.73 & 42 & HN75 & $\mathbf{1 6 . 9 5}$ & $\mathbf{3 5}$ \\
HN124 & 20.71 & 58 & HN116 & $\mathbf{1 6 . 9 3}$ & $\mathbf{4 5}$ \\
HN87 & 20.66 & 77 & HN111 & $\mathbf{1 6 . 9 2}$ & $\mathbf{3 5}$ \\
HN41 & 20.65 & 55 & HN43 & $\mathbf{1 6 . 5 3}$ & $\mathbf{4 2}$ \\
\hline Hold & & & & & \\
\hline
\end{tabular}

Bold font indicates the 20 lines with smaller seeds, and non-bold font indicates the 20 lines with larger seeds.

\section{Population Structure}

Among 109,676 SNPs, we selected 5,000 SNPs with superior polymorphism evenly distributed on the 20 soybean chromosomes. We used STRUCTURE 2.3.4 to calculate $\Delta K$ (Figure 1A; $K=1-10$ ) and identified two subgroups (selected $K=2$ ) based on the $\Delta K$ value (Figure 1B). We analyzed the $r^{2}$ values of all pairs of SNPs located within $10 \mathrm{Mb}$ of each other and determined the LD decay trend based on regression to the negative natural logarithm.

\section{QTNs Detected by Multilocus GWAS Methods}

We detected 23, 32, 9, 20, and 34 significant QTNs using the mrMLM, FASTmrMLM, FASTmrEMMA, pLARmEB, and ISIS EM-BLASSO methods, respectively, with 4, 9, 4, 0, 3, 9, 9, 0, 6, $12,1,6,1,8,5,9,6,14,9$, and 4 significant QTNs detected in each of 20 environments, respectively. No significant QTNs were detected in E4 or E8 (Figure 2 and Supplementary Table S4).

We further examined significant QTNs occurring in multiple environments. Three common QTNs were identified in E2 and E3 (Table 2). AX-157525995 was located on chromosome 6, with LOD values ranging from 3.08 to 4.48 (Table 2). This QTN explained a proportion of phenotypic variance (PVE) of between 3.28 and $6.43 \%$. AX-157369770 was located on chromosome 11, with LOD values ranging from 3.07 to 3.80 (Table 2). This QTN explained a proportion of phenotypic variance (PVE) of between 3.78 and $5.02 \%$ (Table 2). AX-157588392 was located on chromosome 12, with LOD values ranging from 4.20 to 4.26 (Table 2). This QTN explained a proportion of phenotypic variance (PVE) of between 6.36 and $6.64 \%$ (Table 2). The QTN effect direction (positive or negative) was consistent across environments and methods (Table 2).

By comparing the results of different methods, 31 common QTNs were detected by two or more methods (Table 3); these were located on chromosomes $2,3,4,6,7,8,9,10,11,12,14,15$, 16,18 , and 20. Their LOD values ranged from 3.00 to 6.68 , and the PVE ranged from 3.53E-09 to 15.07. The direction of action (positive or negative) for each universal QTN was also consistent across the different methods (Table 3).

Among the 31 common QTNs, 20, 6, 2, and 3 QTNs were detected commonly by two, three, four, and five methods, respectively (Figure 3). From the above studies, mrMLM, FASTmrMLM, and ISIS EM-BLASSO detected a larger number of QTNs among the five methods generally (Figure 4A). In different arrangements of two methods, the number of common QTNs detected by mrMLM combined with FASTmrMLM was large (Figure 4B).

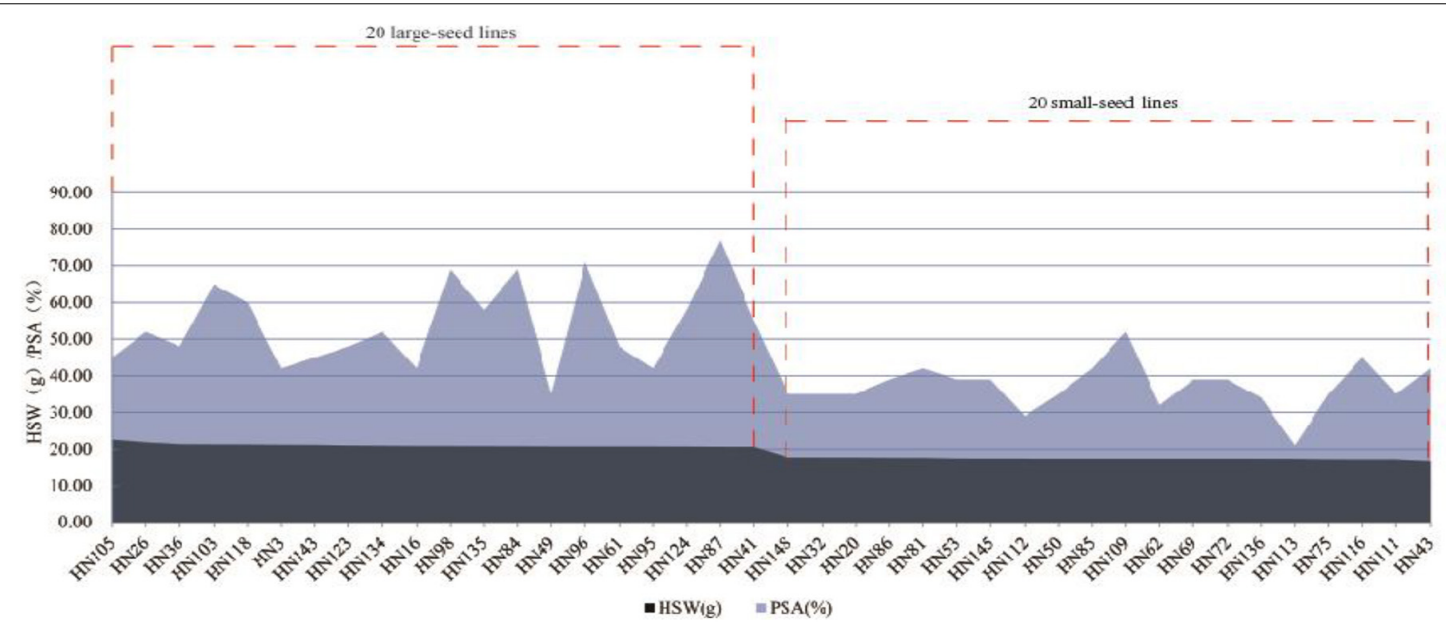

FIGURE 5 | Distribution of superior allele percentages and the hundred-seed weight in the 40 smaller and larger-seed lines. 
TABLE 5 | Superior alleles and their proportions of 31 common QTNs in 20 larger seed lines and 20 smaller seed lines.

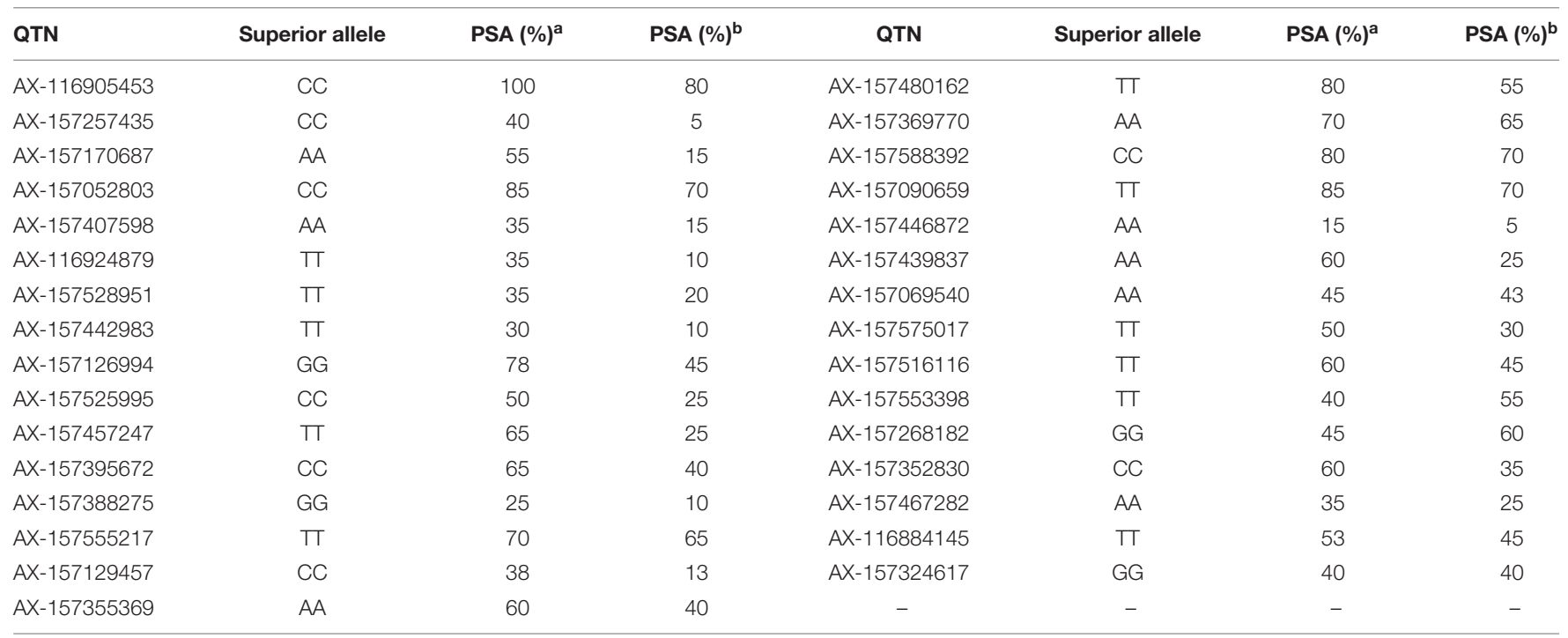

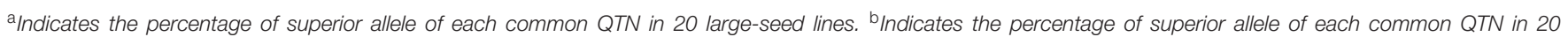
small-seed lines.

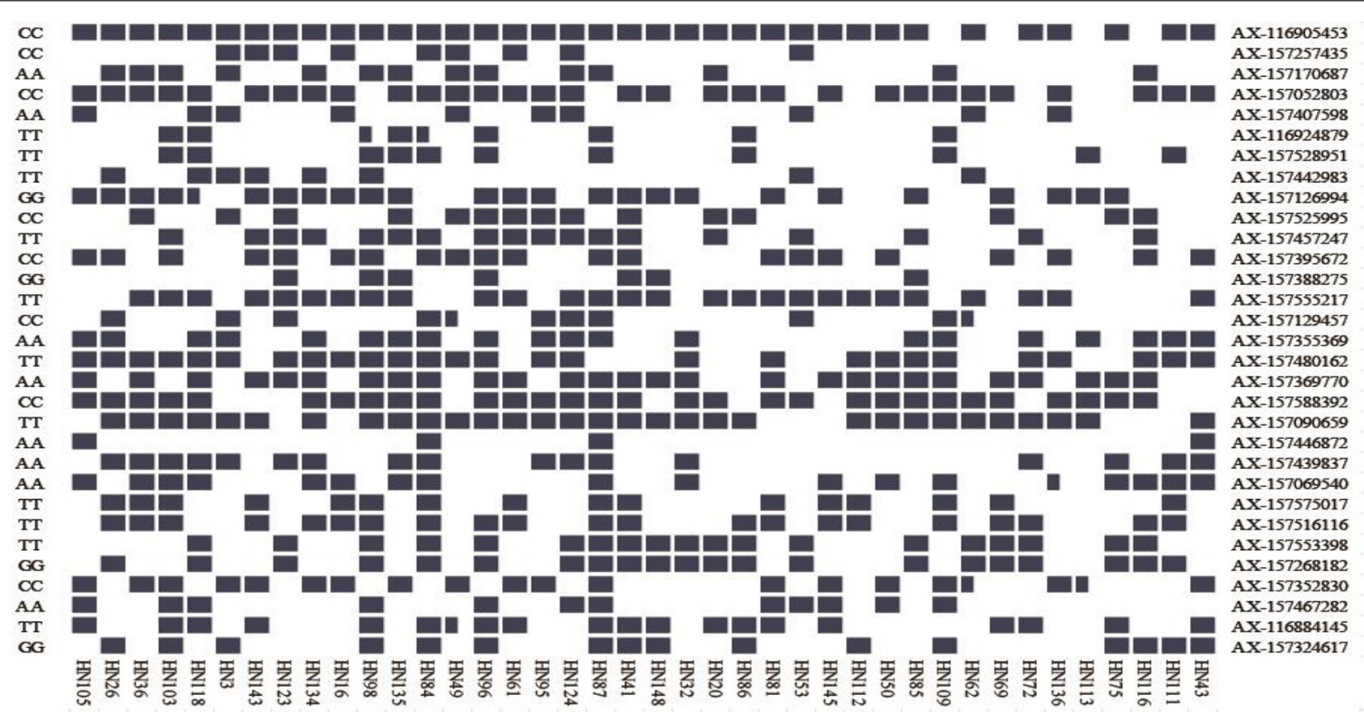

FIGURE 6 | Heat map of the superior allele distribution for the 31 common QTNs in the 40 smaller and larger-seed lines. Blue and white colors represent superior and inferior alleles, respectively.

\section{Distribution of Superior Alleles in the FW-RILs}

We identified 20 large-seed lines with high HSW and 20 smallseed lines with low HSW based on the average phenotypic values of 144 FW-RILs in 20 environments (Table 4). The HSW phenotypic values of the 20 large-seed lines ranged from 20.65 to $22.55 \mathrm{~g}$. For each large-seed line, the percentage of superior alleles (PSAs) for the 31 common QTNs ranged from 35 to $77 \%$; nine lines (45\%) showed a PSA of less than $50 \%$. The phenotypic values for the 20 small-seed lines ranged from 16.53 to $17.79 \mathrm{~g}$, and the PSA ranged from 21 to $52 \%$ (Table 4). Therefore, the large-seed lines had a higher PSA than the small-seed lines (Figure 5).
The PSA range for each common QTN in the 20 large-seed lines was $15-100 \%$, with 18 QTNs having PSAs of $\geq 50 \%$. Among the 20 small-seed lines, PSAs ranged from 5 to $80 \%$, and nine QTNs had PSAs of $>50 \%$ (Table 5 and Figure 6). The number of common QTNs with PSAs $\geq 50 \%$ in 20 large-seed lines was higher than 20 small-seed lines. Based on these results, we can find elite lines by identifying the superior allele ratio of highyield soybeans.

We identified several superior alleles that were present in various large-seed lines; $A X-116905453, A X-157457247, A X-$ 157588392 , and $A X-157090659$ were all present in HN96, HN61, HN95, HN124, HN87, and HN41 (Figure 6). The superior allele 
A

\section{KEGG pathway annotation}

1.Metabolism-

1) Carbohydrate metabolism 2) Energy metabolism 3) Lipid metabolism 4) Nucleotide metabolism

5) Amino acid metabolism

6) Glycan biosynthesis and metabolism

7) Metabolism of cofactors and vitamins 8)Metabolism of terpenoids and polyketides 9) Biosynthesis of other secondary metabolites 2.Genetic Information Processing

\section{1) Transcription}

2) Translation

3) Folding, sorting and degradation 3.Environmental Information Processing-

1) Signal trans duction 4.Cellular Processes-

1) Transport and catabolism

2) Cell growth and death

3) Cellular community - eukaryotes

4) Cellular community - prokaryotes 5.Organismal Systems

1) Immune system

2) Endocrine system

3) Digestive system

4) Nervous system

5) Environmental adaptation 6.Human Diseases

1) Cancers: Overview 2) Cancers: Specific types 3) Immune diseases 4) Substance dependence

5) Cardiovascular diseases 6) Endocrine and metabolic diseases

7) Infectious diseases: Viral

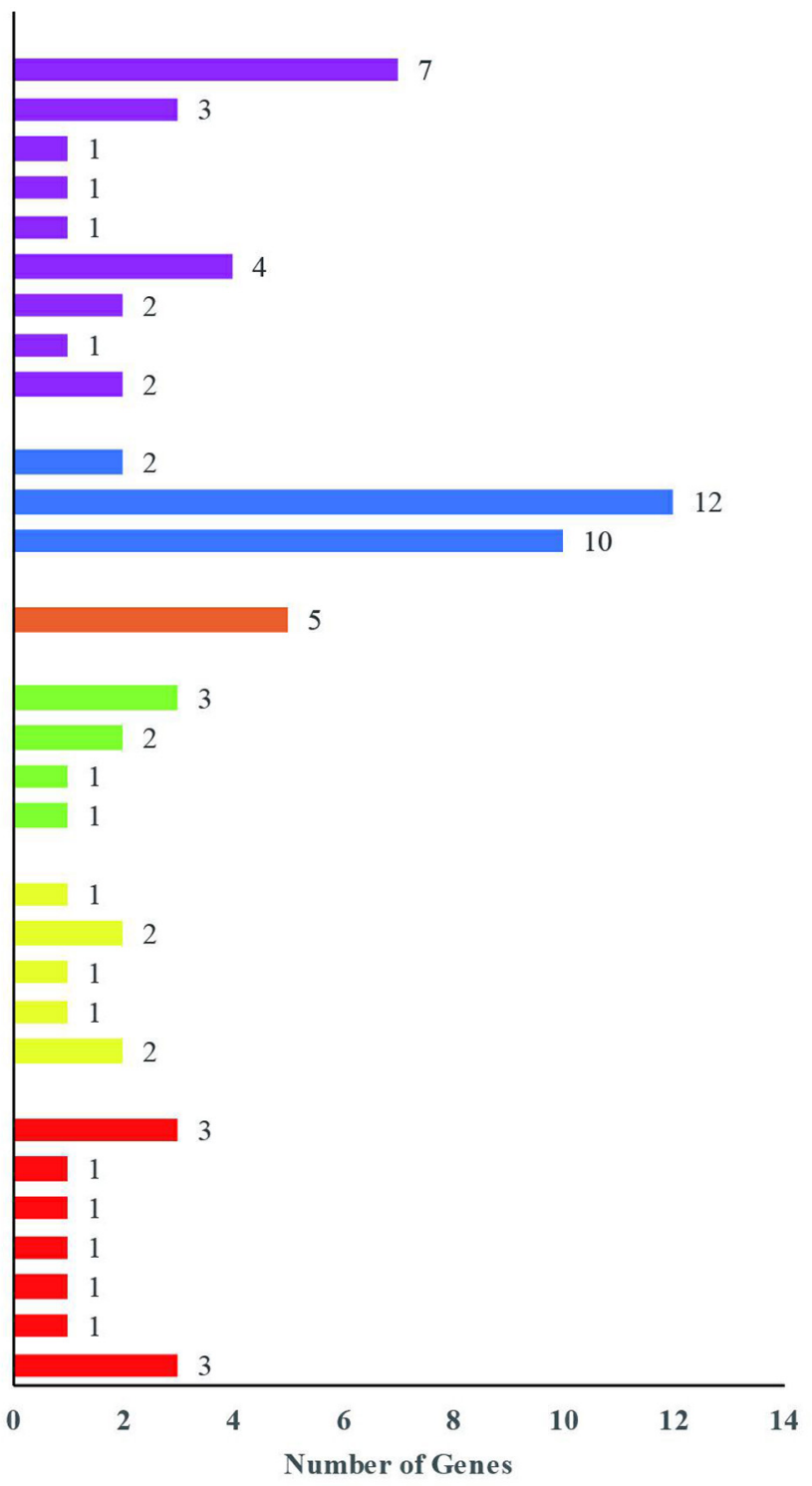

Number of Genes

B

- Protein families: signaling and cellular processes

- Protein families: genetic information processing

- Protein families: metabolism

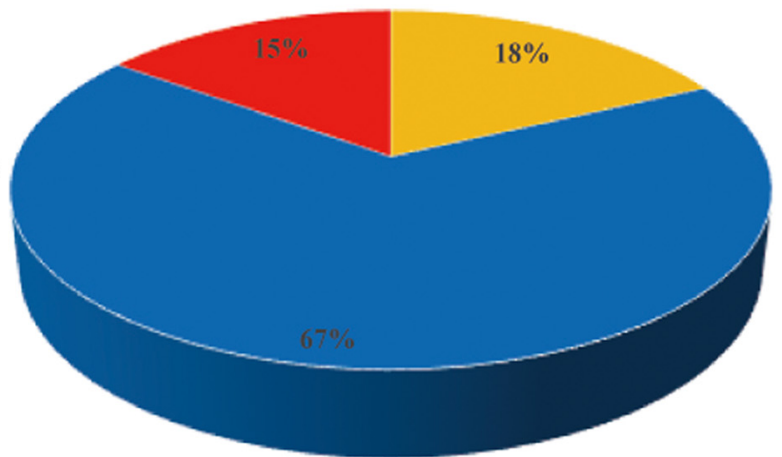

FIGURE 7 | Information on pathways and orthologous protein families of 51 genes. Panel (A) shows the information on pathway. Panel (B) shows the information on orthologous protein families. 
TABLE 6 | Details of three candidate genes annotated in the KEGG database.

\begin{tabular}{|c|c|c|c|c|c|}
\hline QTN name & Gene name $^{a}$ & Chr & Position (bp) & KO number & Annotation \\
\hline$A X-157525995$ & Glyma.06G321900 & 6 & $51040796-51041580$ & K08905 & psaG; photosystem I subunit $\mathrm{V}$ \\
\hline$A X-157555217$ & Glyma.08G365900 & 8 & $47673091-47677260$ & K01809 & manA, MPI; mannose-6-phosphate isomerase [EC:5.3.1.8] \\
\hline$A X-116884145$ & Glyma.20G240000 & 20 & $47137688-47144012$ & K12373 & HEXA_B; hexosaminidase [EC:3.2.1.52] \\
\hline
\end{tabular}

alndicates the gene which correlates with the QTN.

AX-116905453 was present in all 20 large-seed lines. We believe that superior alleles may have an important effect on the HSW of soybeans. In further research, we hope to use this information to develop larger seed size soybean varieties through markerassisted selection.

\section{Potential Candidate Genes Determined Based on Common QTNs}

For each common QTN, we use the LD decay distance as the interval range to find candidate genes. Because the population we selected was not a natural group, but an FW-RIL population, the LD decay distance was very large. We therefore chose the range of potential candidate genes according to the location with the largest decay rate. As the LD decayed fastest before $200 \mathrm{~kb}$ and then tended to flatten (Figure 2A), we searched for potential candidate genes at $100-\mathrm{kb}$ intervals on either side of each QTN. We identified 635 genes in this interval, of which 129 were highly expressed during seed formation (Supplementary Table S7). Based on the annotation data, 51 of the 129 genes were annotated in 29 pathways and three protein families in the KEGG database (Supplementary Tables S5, S6 and Figure 7). Three of these are potential candidate genes based on their annotation information and function in metabolic pathways (Table 6).

\section{DISCUSSION}

Most genetic analyses of yield-related traits in soybean, such as HSW, have been based on RILs arising from the hybridization of two parents used for QTL mapping analyses. Sites detected in these analyses will therefore be suitable only for that specific population. Most mapping populations involve a limited number of recombination, and few molecular markers can be used; fine mapping is challenging. Four-way RILs were derived from four parents, and there may be one to four alleles in a QTL. As long as there is a significant difference in genetic effect between the two alleles in a QTL, this QTL is detected. Therefore, the intensity of QTL detection and superior allele selection are improved. In recent years, with the rapid development of GWAS, natural population is generally used as research materials to identify HSW QTL in soybean. The FW-RILs used in this study are the MAGIC (multiparent advanced generation intercross) lines, which were artificially configured with good linkage relationship, whereas the natural population has no linkage relationship. The number of parents of the FW-RILs is limited, and the population structure is not obvious compared with the natural population composed of many germplasm resources. Thus, the false-positive rate of FW-RILs is lower than that of the natural population; the statistical power and QTL positioning accuracy are higher.
Previous studies have shown that HSW is a typical quantitative trait controlled by multiple genes, and it is controlled by many microgene loci under different genetic conditions. A GWAS using 31,283 SNPs and CMLM detected five loci associated with HSW (Copley et al., 2018). Yan et al. (2017) used SoySNP50K BeadChip to genotype more than 42,000 SNPs, and the MLM accounting for both population structure and kinship was conducted for GWAS. Eight SNPs located on chromosomes 4 and 17 were significantly associated with HSW. The variation explained by significant markers $\left(R^{2}\right)$ ranged from 6.9 to $13.2 \%$. Contreras-Sota et al. (2017) used SNP markers and MLM considering Q + K models for GWAS to identify genomic regions controlling HSW. Seven SNPs were significantly associated with HSW on chromosomes 5, 7, 11, and 12 across the locations under study, and $R^{2}$ ranged from 13.2 to $31.2 \%$. Although high-density SNP markers were applied in these experiments, statistical methods (Yu et al., 2006) treating single loci mainly detect QTLs with high heritability and neglect those with moderate or low heritability. The five multilocus GWAS methods used in this study greatly reduce the false positives in the result and increase the credibility of the results. All potential QTNs with large or small effects in different statistical models could be detected by the methods.

We performed a multilocus genome-wide association analysis of HSW in soybean using a high-density SNP chip and mapped 31 common QTNs in 20 environments (Table 3). Currently, the SoyBase database includes 314 targeted HSW QTLs ${ }^{1}$. The 11 common QTNs identified in this study were present in the SoyBase database: $A X-157439837, A X-157129457$, $A X-157090659, A X-157467282, A X-157324617, A X-157407598$, $A X-157369770, A X-157528951, A X-157388275, A X-157442983$, and $A X-157257435$. Quantitative trait loci physically close to these were identified previously (Mian et al., 1996; Hyten et al., 2004; Chen et al., 2007; Li D. et al., 2008; Teng et al., 2009; Liu et al., 2011; Han et al., 2012; Kato et al., 2014; Gai et al., 2016; Hacisalihoglu et al., 2018). This supports the accuracy of our study and validates the authenticity of the loci involved in seed size formation.

Based on the common QTNs detected in this study and the annotated pathways associated with these QTNs, we identified three genes that may be associated with HSW in soybean (Table 6). Glyma.06G321900 is associated with psaG, an intrinsic membrane protein associated with Photosystem I (PSI). psaG is a nuclear-encoded gene corresponding to PSI subunit V of spinach (Steppuhn et al., 1988). Further experiments evaluate the potential role of subunit $\mathrm{V}$ in PSI

${ }^{1}$ http://www.soybase.org/ 
function under water-deficit stress (Wood and Duff, 1999). Photosynthesis converts light energy into chemical energy for plant growth, increasing crop yield. Therefore, we speculate that this gene increases photosynthesis in the absence of water, which indirectly affects the HSW of soybean. Glyma.08G365900 is related to mannose phosphate isomerase (PMI), and the PMI (manA) gene encodes a mannose-6-phosphate transferase, catalyzing the conversion of 6-phosphoric acid mannose to fructose 6-phosphate. Cells can metabolize fructose via the glycolytic pathway (Reed et al., 2001), rendering mannose a carbon source for the growth of transformed cells, and thus the plant exhibits a more prominent growth advantage. We speculate that PMI (manA) indirectly controls the HSW of soybean plants. Glyma.20G240000 is associated with hexosaminidase (Hex). During fruit ripening, $\beta$-Hex releases a large amount of free $N$-glycans from glycoprotein polypeptide chains (Yunovitz and Gross, 1994). Free $N$-glycans are important signaling molecules that promote fruit ripening (Handa et al., 1985; Priem and Gross, 1992), possibly through $\beta$-Hex triggering ethylene synthesis systems (Jagadeesh et al., 2004). We therefore suggest that Hex controls soybean seed development by affecting the biosynthesis of phytohormones.

\section{Summary}

We identified 31 QTNs using a five-multilocus GWAS method. An analysis of the common QTNs identified three candidate genes. By analyzing the ratio of superior alleles in large- and small-seed lines, we established that the superior alleles of these common QTNs might be useful for molecular marker-assisted breeding of soybean plants with larger seeds.

\section{REFERENCES}

Belamkar, V., Farmer, A. D., Weeks, N. T., Kalberer, S. R., Blackmon, W. J., and Cannon, S. B. (2016). Genomics-assisted characterization of a breedingcollection of Apios americana, an edible tuberous legume. Sci. Rep. 6:34908. doi: 10.1038/srep34908

Bradbury, P. J., Zhang, Z., Kroon, D. E., Casstevens, T. M., Ramdoss, Y., and Buckler, E. S. (2007). TASSEL: software for association mapping of complex traits in diverse samples. Bioinformatics 23, 2633-2635. doi: 10.1093/ bioinformatics/btm 308

Chen, Q. S., Zhang, Z. C., Liu, C. Y., Xin, D. W., Shan, D. P., Qiu, H. M., et al. (2007). QTL analysis of major agronomic traits in soybean. Sci. Agric. Sin. 40, 41-47.

Contreras-Sota, I. R., Mora, F., Mar, O., Higashi, W., and Schuster, I. (2017). A genome-wide association study for agronomic traits in soybean using snp markers and snp-based haplotype analysis. PLoS One 12:e0171105. doi: 10.1371/ journal.pone.0171105

Copley, T. R., Duceppe, M. O., O’ Donoughue, U., and Louise, S. (2018). Identification of novel loci associated with maturity and yield traits in early maturity soybean plant introduction lines. BMC Genom. 19:167. doi: 10.1186/ s12864-018-4558-4554

Doyle, J. J., Doyle, J. L., and Brown, A. H. D. (1990). Analysis of a polyploid complexin Glycine with chloroplast and nuclear DNA. Austr. Syst. Bot. 3, 125-136. doi: 10.1071/SB9900125

Earl, D. A., and Vonholdt, B. M. (2012). Structure harvester: a website and program for visualizing STRUCTURE output and implementing the evanno method. Conserv. Genet. Resour. 4, 359-361. doi: 10.1007/s12686-0119548-7

\section{DATA AVAILABILITY STATEMENT}

The genotyping data is available at https://figshare.com/s/ 84eb97ad5c5e523072e5.

\section{AUTHOR CONTRIBUTIONS}

WL and HN conceived and designed the experiments. JS, XT, YW, YF, XL, JW, CY, SJ, and XS performed the field experiments. SL and ZT performed the genome sequencing. ZQ, KZ, and $\mathrm{HN}$ analyzed and interpreted the results. ZQ and $\mathrm{HN}$ drafted the manuscript and all authors contributed to manuscript revision. WL acquired the funding. All authors contributed to the article and approved the submitted version.

\section{ACKNOWLEDGMENTS}

The authors gratefully acknowledge financial support for this study provided by grants from the National Key Research and Development Program of China (2017YFD0101303-6) to WL.

\section{SUPPLEMENTARY MATERIAL}

The Supplementary Material for this article can be found online at: https://www.frontiersin.org/articles/10.3389/fgene. 2020.00689/full\#supplementary-material

Evanno, G., Regnaut, S., and Goudet, J. (2005). Detecting the number of clusters of individuals using the software structure: a simulation study. Mol. Ecol. 14, 2611-2620. doi: 10.1111/j.1365-294x.2005.02553.x

Fang, C., Ma, Y., Wu, S., Liu, Z., Wang, Z., Yang, R., et al. (2017). Genome-wide association studies dissect the genetic networks underlying agronomical traits in soybean. Genome Biol. 18:161. doi: 10.1186/s13059-017-1289-9

Gai, J. Y., Wang, Y. J., Wu, X. L., and Chen, S. (2016). A comparative study on segregation analysis and qtl mapping of quantitative traits in plantswith a case in soybean. Front. Agric. China 1, 1-7. doi: 10.1007/s11703-0070001-3

Hacisalihoglu, G., Burton, A. L., Gustin, J. L., Eker, S., Asikli, S., Heybet, E. H., et al. (2018). Quantitative trait loci associated with soybean seed weight and composition under different phosphorus levels. J. Integr. Plant Biol. 60, 232241. doi: $10.1111 /$ jipb. 12612

Han, Y., Li, D., Zhu, D., Li, H., Li, X., Teng, W., et al. (2012). QTL analysis of soybean seed weight across multi-genetic backgrounds and environments. Theor. Appl. Genet. 125, 671-683. doi: 10.1007/s00122-012-1859-x

Handa, A. K., Singh, N. K., and Biggs, M. S. (1985). Effect of tunicamycin on in vitro ripening of tomato pericarp tissue. Physiol. Plant. 63, 417-424. doi: 10.1111/j.1399-3054.1985.tb02320.x

He, L. Q., Xiao, J., Rashid, K. Y., Yao, Z., Li, P., Jia, G., et al. (2019). Genome-wide association studies for pasmo resistance in flax (Linum usitatissimum 1.). Front. Plant Sci. 9:1982. doi: 10.3389/fpls.2018.01982

Hyten, D. L., Pantalone, V. R., Sams, C. E., Saxton, A. M., Landau-Ellis, D., Stefaniak, T. R., et al. (2004). Seed quality QTL in a prominent soybean population. Theor. Appl. Genet. 109, 552-561. doi: 10.1007/s00122-004-1661-5

Jagadeesh, B. H., Prabha, T. N., and Srinivasan, K. (2004). Activities of glycosidases during fruit development and ripening of tomato (Lycopersicum esculantum L.): 
implication in fruit ripening. Plant Sci. 166, 1451-1459. doi: 10.1016/j.plantsci. 2004.01.028

Kang, H. M., Zaitlen, N. A., Wade, C. M., Kirby, A., Heckerman, D., Daly, M. J., et al. (2008). Efficient control of population structure in model organism association mapping. Genetics 178, 1709-1723. doi: 10.1534/genetics. 107.080101

Kato, S., Sayama, T., Fujii, K., Yumoto, S., Kono, Y., Hwang, T.-Y., et al. (2014). A major and stable QTL associated with seed weight in soybean across multiple environments and genetic backgrounds. Theoret. Appl. Genet. 127, 1365-1374. doi: 10.1007/s00122-014-2304-2300

Lander, E. S., and Botstein, D. (1989). Mapping mendelian factors underlying quantitative traits using rflp linkage maps. Genetics 121, 185-189. doi: 10.1007/ BF00121515

Li, D., Pfeiffer, T. W., and Cornelius, P. L. (2008). Soybean QTL for yield and yield components associated with alleles. Crop Sci. 48:571. doi: 10.2135/cropsci2007. 06.0361

Li, W. X., Zheng, D. H., Van, K., and Lee, S.-H. (2008). QTL mapping for major agronomic traits across two years in soybean (Glycine max L. Merr.). J. Crop Sci. Biotechnol. 11, 171-176.

Liu, W., Lee, S.-H., Kim, M. Y., Van, K., Lee, Y.-H., Li, H., et al. (2011). QTL identification of yield-related traits and their association with flowering and maturity in soybean. J. Crop Sci. Biotechnol. 14, 65-70. doi: 10.1007/s12892010-0115-7

Mellbye, B., and Schuster, M. (2014). Physiological framework for the regulation of quorum sensing-dependent public goods in Pseudomonas aeruginosa. Bacteriology 196, 1155-1164. doi: 10.1128/JB.01223-13

Mian, M. A. R., Bailey, M. A., Tamulonis, J. P., Shipe, E. R., Carter, T. E. Jr., Parrott, W. A., et al. (1996). Molecular markers associated with seed weight in two soybean populations. Theoret. Appl. Genet. 93, 1011-1016. doi: 10.1007/ bf00230118

Priem, B., and Gross, K. C. (1992). Mannosyl- and Xylosyl-containing glycans promote tomato (Lycopersicon esculentum Mill.) fruit ripening. Plant Physiol. 98, 399-401. doi: 10.1104/pp.98.1.399

Pritchard, J. K., Stephens, M., Rosenberg, N. A., and Donnelly, P. (2000). Association mapping in structured populations. Am. J. Hum. Genet. 67, 170181. doi: 10.1086/302959

Reed, J., Privalle, L., Powell, M. L., Meghji, M., and Dawson, J. (2001). Phosphomannose isomerase: an efficient selectable marker for plant transformation. Vitro Cell. Dev. Biol. Plant 37, 127-132. doi: 10.1007/s11627-001-0024-z

Reinprecht, Y., Poysa, V. W., Yu, K., Rajcan, I., Ablett, G. R., and Pauls, K. P. (2006). Seed and agronomic QTL in low linolenic acid, lipoxygenase-free soybean (Glycine max (L.) Merrill) germplasm. Genome 49, 1510-1527. doi: 10.1139/ g06- 112

Steppuhn, J., Hermans, J., Nechushtai, R., Ljungberg, U., Thummler, F., Lottspeich, F., et al. (1988). Nucleotide sequence of cDNA clones encoding the entire precursor polypeptides for subunits IV and $\mathrm{V}$ of the photosystem I reaction center from spinach. FEBS Lett. 237, 218-224. doi: 10.1016/0014-5793(88) 80205-80209

Tamba, C. L., Ni, Y. L., and Zhang, Y. M. (2017). Iterative sure independence screening EM-Bayesian LASSO algorithm for multi-locus genome-wide association studies. PLoS Comput. Biol. 13:e1005357. doi: 10.1371/journal.pcbi. 1005357

Tamba, C. L., and Zhang, Y. M. (2018). A fast mrMLM algorithm for multi-locus genome-wide association studies. bioRxiv [Preprint], doi: 10.1101/341784

Teng, W., Han, Y., Du, Y., Sun, D., Zhang, Z., Qiu, L., et al. (2009). QTL analyses of seed weight during the development of soybean (Glycine max L. Merr.). Heredity 102, 372-380. doi: 10.1038/hdy.2008.108

Wang, J. K. (2009). The complete range of quantitative character gene mapping method. J. Crops 35, 239-245. doi: 10.3724/SP.J.1006.2009.00239

Wang, S. B., Feng, J. Y., Ren, W. L., Huang, B., Zhou, L., Wen, Y., et al. (2016a). Improving power and accuracy of genome-wide association studies via a multi-locus mixed linear model methodology. Sci. Rep. 6:19444. doi: 10.1038/ srep 19444

Wang, S. B., Wen, Y. J., Ren, W. L., Ni, Y. L., Zhang, J., Feng, J. Y., et al. (2016b). Mapping small-effect and linked quantitative trait loci for complex traits in backcross or DH populations via a multi-locus GWAS methodology. Sci. Rep. 6:29951. doi: 10.1038/srep29951

Wang, X., Xu, Y., Li, G. J., Li, H. N., Gen, W. Q., and Zhang, Q. M. (2010). Identify QTL associated with soybean 100-seed weight. Science 36, 1674-1682. doi: 10.3724/SP.J.1006.2010.01674

Wen, Y. J., Zhang, H. W., Ni, Y. L., Huang, B., Zhang, J., Feng, J. Y., et al. (2017). Methodological implementation of mixed linear models in multi-locus genome-wide association studies. Brief. Bioinform. 19, 700-712. doi: 10.1093/ bib/bbw 145

Wen, Y. J., Zhang, Y. W., Zhang, J., Feng, J. Y., Jim, M. D., and Zhang, Y. M. (2018). An efficient multi-locus mixed model framework for the detection of small and linked QTLs in F2. Brief. Bioinform. 20, 1-12. doi: 10.1093/bib/ bby 058

Wood, A. J., and Duff, R. J. (1999). Subunit V (PsaG) of the photosystem i reaction center (accession No. AF157017) from desiccated Tortula ruralis. Plant Physiol. $121,313$.

Yan, L., Hofmann, N., Li, S. X., Ferreira, M. E., Song, B. H., Jiang, G. L., et al. (2017). Identification of QTL with large effect on seed weight in a selective population of soybean with genome-wide association and fixation index analyses. BMC Genom. 18:29. doi: 10.1186/s12864-017-3922-0

Yu, J. M., Pressoir, G., Briggs, W. H., Bi, I. V., Yamasaki, M., Doebley, J. F., et al. (2006). A unified mixed-model method for association mapping that accounts for multiple levels of relatedness. Nat. Genet. 38, 203-208. doi: 10.1038/ng1702

Yunovitz, H., and Gross, K. C. (1994). Effect of tunicamycin on metabolism of unconjugated $\mathrm{N}$-glycans in relation to regulation of tomato fruit ripening. Phytochemistry 37, 663-668. doi: 10.1016/s0031-9422(00)90334-0

Zeng, Z. B. (1994). Precision mapping of quantitative trait loci. Genetics 136, 1457-1468. doi: 10.1007/s00122-012-2032-2

Zhang, J., Feng, J. Y., Ni, Y. L., Wen, Y. J., Niu, Y., Tamba, C. L., et al. (2017). pLARmEB: integration of least angle regression with empirical Bayes for multilocus genome-wide association studies. Heredity 118, 517-524. doi: 10.1038/ hdy. 2017.8

Zhang, Y., Ge, F., Hou, F., Sun, W., Zheng, Q., Zhang, X., et al. (2017). Transcription factors responding to $\mathrm{Pb}$ stress in maize. Genes 8:E231. doi: 10.3390/genes8090231

Zhang, J. P., Song, Q. J., Cregan, P. B., and Jiang, G. L. (2016). Genome-wide association study, genomic prediction and marker-assisted selection for seed weight in soybean(Glycine max). Theoret. Appl. Genet. 129, 117-130. doi: 10. 1007/s00122-015-2614-x

Zhang, Z., Ersoz, E., Lai, C.-Q., Todhunter, R. J., Tiwari, H. K., Gore, M. A., et al. (2010). Mixed linear model approach adapted for genome-wide association studies. Nat. Genet. 42, 355-360. doi: 10.1038/ng.546

Zhou, Z. K., Jiang, Y., Wang, Z., Gou, Z. H., Lyu, J., Li, W. Y., et al. (2015). Resequencing 302 wild and cultivated accessions identifies genes related to domestication and improvement in soybean. Nat. Biotechnol. 33, 408-414. doi: $10.1038 /$ nbt.3096

Conflict of Interest: The authors declare that the research was conducted in the absence of any commercial or financial relationships that could be construed as a potential conflict of interest.

Copyright (C) 2020 Qi, Song, Zhang, Liu, Tian, Wang, Fang, Li, Wang, Yang, Jiang, Sun, Tian, Li and Ning. This is an open-access article distributed under the terms of the Creative Commons Attribution License (CC BY). The use, distribution or reproduction in other forums is permitted, provided the original author(s) and the copyright owner(s) are credited and that the original publication in this journal is cited, in accordance with accepted academic practice. No use, distribution or reproduction is permitted which does not comply with these terms. 